\title{
Steps Towards Online Monitoring Systems and Interoperability
}

\author{
Diana Ferreira ${ }^{1}$, Cristiana Neto ${ }^{1}$, José Machado ${ }^{2}$ and António Abelha ${ }^{2}$ \\ ${ }^{1}$ Informatics Department, University of Minho, Braga, Portugal \\ ${ }^{2}$ Algoritmi Research Center, University of Minho, Braga, Portugal \\ \{a72226, a72064\}ealunos.uminho.pt, \\ \{jmac, abelha\}@di.uminho.pt
}

\begin{abstract}
In the health area, there is, on a daily basis, an enormous amount of data being produced and disseminated. The fast-growing amount of collected data and the rich knowledge, possibly life-saving, that could be extracted from these data has demanded the search of new ways to ensure the reliability and availability of the information with an emphasis on the efficient use of information technology tools. Although the main focus of the information systems is the health professionals who contact directly with the patient, it is also imperative to have tools for the background of the health units (information services, managers of systems, etc.). The main purpose of this work is the development of an innovative and interactive web platform for the daily monitoring of the web services activities of a Portuguese hospital, Centro Hospitalar do Porto (CHP). This platform is a web application developed in React that aims to ensure the correct functioning of the web services, that are responsible for numerous tasks within the hospital environment, and which failure could result in disastrous consequences, both for the health institution and for the patients. The development of the web application followed the six stages of the Design Science Research (DSR) methodology and was submitted to the Strengths Weaknesses Opportunities and Threats (SWOT) analysis, which results were considered optimistic.
\end{abstract}

Keywords: Information Technology, Monitoring, Web Services, Web Application.

\section{Introduction}

The last years have been critical for the foundation of a new world-wide era, being particularly remarkable the influence of a diverse set of forces and tendencies associated with the acceleration of the scientific and technological progress in the information domain. The "Information Era" promotes, in a global context of increasing dynamism, a permanent revolution of knowledge and perceptions in practically all areas of human knowledge. Information is a decisive factor in the survival of any organization. An information system (IS) is a socio-technical subsystem of an organization that collects, stores, processes, transmits and displays data, information, and knowledge relevant to it [1][2][3][4]. This meets Davis \& Olson's (1985) vision of an IS, which is an integrated man/machine system that provides information to support operations and decision-making in organizations [5]. 
Information is especially crucial to the health organizations, either for its action, i.e., its decision-making process, or for its reaction, i.e., the control and correction of deviations from its action. The information technologies have the potential to ensure the efficient delivery of health care and improve the quality of services provided by health professionals as they provide comprehensive and credible information and support the decision-making process, both clinically and administratively, thereby reducing the incidence of adverse events and clinical errors [6][7][8].

There is a whole set of economic, technological, social and political factors that impel health organizations to search for new ways to improve the quality of the services provided. Faced with this reality, these organizations should be prepared to make more informed and less intuitive decisions in the shortest time possible. Accordingly, health institutions have been adopting health information systems (HISs). HISs are complex socio-technical subsystems processing data, information, and knowledge to ease the management of clinical and administrative information and the planning, refinement and decision-making process of the different professionals of the health system [1][9]. Since the main focus of a hospital is the patients' health status, most of these systems are dedicated to the diagnosis, treatment, and follow-up of patients. However, it is necessary to make decisions related to the computer processes of the hospital, such as web services, which also, indirectly, influence the quality of care. Therefore, the present study focuses on the development of an innovative and interactive web platform for the daily monitoring of the web services activities of the CHP.

The presented paper is organized as follows: section II depicts the different stages of the DSR methodology that led to the development of the web platform, section III refers to the discussion of the results achieved, and section IV discloses the main conclusions outlined with this work.

\section{Monitoring Platform for Web Services Activity}

A health institution must continuously monitor the technological solutions implemented and used in its hospital units. Monitoring means overseeing ongoing activities to ensure that they meet the standards and purposes established in the initial projection. It is a continuous process, consisting of three components: data collection, regular analysis of data and dissemination of data to all stakeholders. This definition can be adapted to the computing world by using the available electronic means for such monitoring. Regarding the health units' computer systems, monitoring involves evaluating the performance of services provided by computers or communication systems.

The main purpose of this project was to monitor the activities of the web services of the CHP through a monitoring platform, that is, a web application using Business Intelligence (BI) technology, in order to better control the aspects that condition the web services performance, using the periodic collection of data and the analysis of indicators. These indicators are parameters that should help to summarize and understand important factors related to the data, such as the number of errors reported in a certain period of time. In this way, it is possible to discover a potential common cause for the occurrence of errors and to plan and implement specific and efficient measures 
to reduce the incidence of errors in these critical operations, increasing the quality of health care delivery.

The BI technology is used to generate and present these parameters, as it allows the processing of data and the analysis of the information extracted from these data, in an easy and efficient way.

The web application was developed based on the DSR methodology (see Fig. 1). The first steps of this methodology concern the identification of a problem that needs to be solved and the definition of the objectives of the possible solution. All the subsequent phases aim to develop and evaluate the proposed solution. Accordingly, the following subsections contain the description of the web application development process and are organized according to the six phases of the DSR methodology.

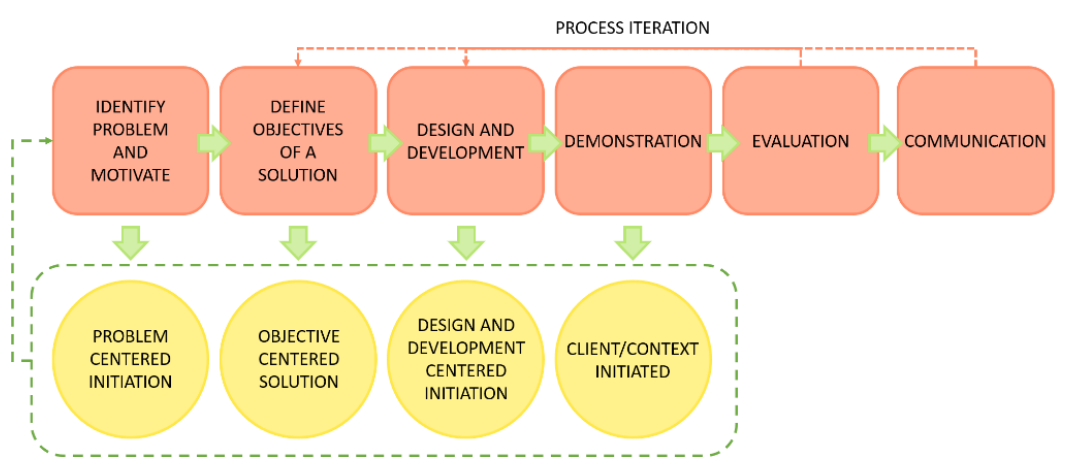

Fig. 1. Model of the DSR methodology. Adapted from [10].

\subsection{Problem identification and motivation}

In the first stage it was made an overview of the current state of the information systems of the CHP, in order to find problems and possible areas of intervention. There are two needs at the CHP that should be eliminated. Firstly, the need of a system to visualize various aspects of the activities of the web services was identified. Secondly, it is necessary to manage this activity and evaluate its behavior over time allowing a quick perception of performance problems and thus decreasing the time of its resolution. Hence, the present work arises from the need to continuously monitor the hospital environment as well as the operations performed in it and the results of its activities in order to understand if the web services are functional and if its performance meets the defined standards, in addition to timely notice the occurrence of errors to apply specific measures in the moment.

The core motivation is related to the need for managers to make fast and informed decisions in order to improve the productivity and efficiency of the organization and, at the same time, the quality of the care provided. There is an imperative need to simplify the presentation and analysis of information regarding the activity of the web services, in order to assist professionals in making decisions related to the control of these activities and the prevention of the occurrence of errors in the web services. 


\subsection{Objectives definition}

After the definition of the problem and the explanation of the surrounding motivations, all the objectives to be fulfilled were outlined. The main purpose of this project is to provide a dynamic, interactive, and user-friendly platform to allow key management staff to consult and manage the web services' performance in order to keep track of the hospital's operations and study the processes behavior optimizing them and improving its operational performance. In addition, some adjacent objectives were outlined:

- Collection of information related to the activity of the web services;

- Study of technical requirements necessary in the design and development of the monitoring platform;

- Decide what the best type of web service is to work as a basis for interoperability between the hospital databases and the platform developed, according to the needs and the means available;

- Creation of a user-friendly platform in React with the following purposes:

- Gathering reliable summary data and providing key management staff greater visibility into the information required to make critical decisions and collect greater insights for the web service's performance indicators;

- Identification of a set of features concerning targets, purposes and goals, data sources, indicators and their graphical representation, analytics support, and data processing approaches;

- Creation of visual interacting live dashboards that enable the monitorization of hospital's operations and the study of processes behavior in order to optimize them and improve its operational performance.

- Maintenance of the proper functioning of the web services in order to ensure that the solutions satisfy the requirements for which they were developed.

\subsection{Design and Development}

Frameworks and Languages. The information generated and presented to the user in the form of a monitoring system contains BI modules, and basic information presentation structures (tables). To collect this data, a visual basic .NET function was created to obtain information about the web services' activities. This function is called in several phases of the web service execution, to ensure that the information regarding the occurrence of errors is obtained in all possible places for these errors to occur. It also allows to get the time when the web service was first called and the end time of its execution in order to be able to obtain the duration of execution of the web service and consequently to control and optimize it whenever necessary. This information was stored in a new table that was added to the hospital's SIL database. 
This monitoring platform is, in fact, a web application that was developed using ReactJS, a JavaScript library for building user interfaces. PHP and SQL programming languages were also used, but to develop RESTful PHP Web services and query requests for Oracle databases, respectively. JavaScript executes HTTP requests to a server of the type REST implemented in the PHP language which constitutes the backend of the application, to make the data available. The data is selected through SQL queries and sent in the JSON format. Finally, Apache corresponds to the Web server that supports the Web application.

REST api is a need because it is the lightest way to create, read, update or delete information between different applications over the internet or HTTP protocol. PHP was the programming language used to build the RESTFUL server because of its easy implementation and interpretation using Apache.

It is extremely important to take into consideration the visual aspect of the application, as in certain cases it is directly related to the non-adoption of those applications. This is the main reason why this web application was developed in React, as it is a modern library that is taking over the front-end development.

Fig. 2 shows the entire architecture of this web application, including its components and their interactions.

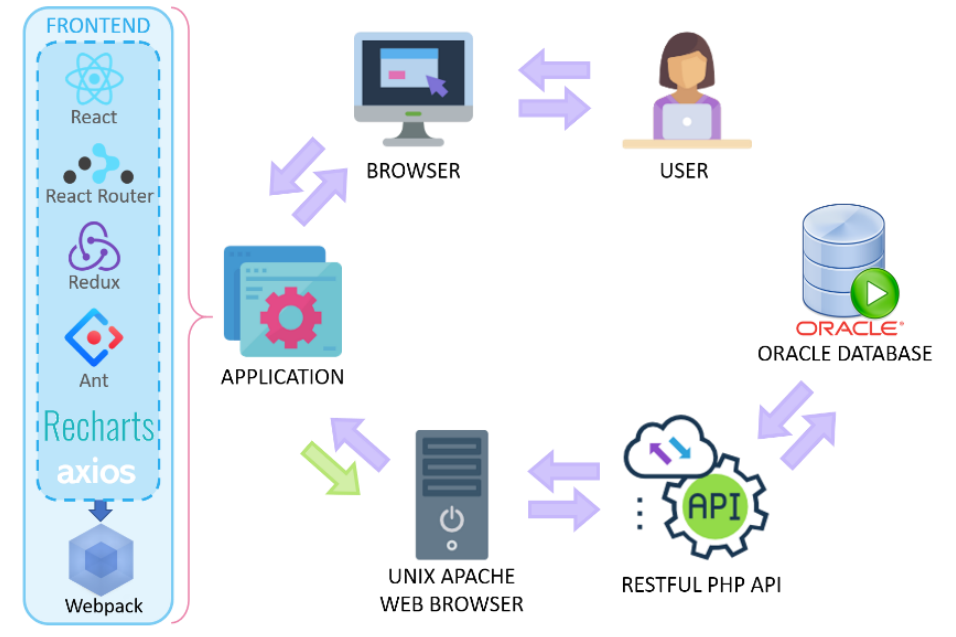

Fig. 2. Web application architecture.

Main modules. Regarding the design of the interface, the monitoring platform has a side menu which grants access to three distinct modules, namely the Dashboards Module, Indicators Module, and Tables Module. The information provided could have been displayed in just one single page. However, it was unclear, overwhelming and confusing to the user. Accordingly, this decision was made to organize and present information in a way that is easy to read. Fig. 3 shows the visual aspect of the web application interface. 


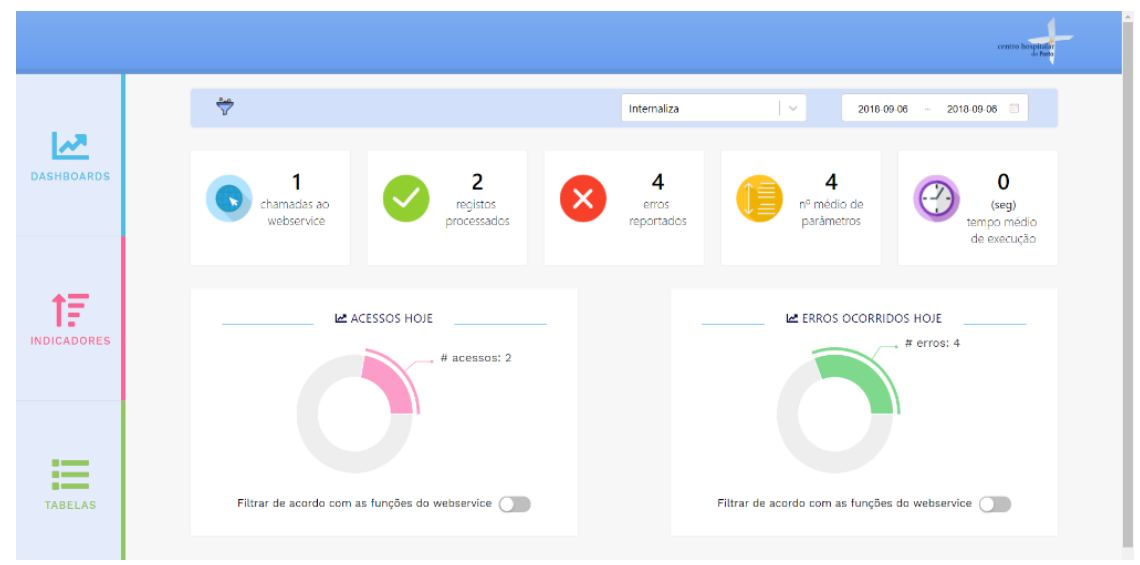

Fig. 3. Overview of the web application interface.

It is important to note that the charts presented in the web application were built using the Recharts library that facilitates the construction of charts in React since it results from the combination of React and D3. Next, each module of the web application will be described.

Dashboards Module. Presents interactive dashboards, according to the selected filters. These filters concern the choice of the web service on which it is intended to analyze information about its activity and the choice of the time interval that limits this information. When clicking the dates filter, a calendar appears that allows the selection of any range of dates, and below, easy access options are available with some common date range options, like today or last week. This page presents some relevant statistical values, like the number of calls made to the web service and the average time of execution of that web service. Immediately next to the above statistics, two graphs are presented with information about the activity of the web services, one corresponding to the accesses to the web service and the other one related to the errors occurred in it. It is important to note that each of these graphs presents the possibility of filtering according to the methods of the web service, i.e., it allows to separate the data visualized according to the different methods that constitute the web service.

Indicators Module. Contains all the performance indicators of the web services considered relevant and important when monitoring its activities. These indicators were defined according to the manager's needs. After being gathered and developed, these indicators were analyzed, refined and checked for completeness. The final indicators were:

- Number of accesses to the web service in specific dates, like, today, last week and last year;

- Number of errors occurred in the web service in specific dates, like, today, last week and last year;

- Number of accesses to the web service per year; 
- Number of accesses to the web service per month;

- Number of errors occurred in the web service per year;

- Number of errors occurred in the web service per month;

- Number of accesses to the web service according to its different methods;

- Number of errors occurred in the web service according to its different methods.

To ease the visualization and understanding of the indicators related to the number of accesses and errors by a specific date, it was decided to group them all on a chart (see Fig. 4). These indicators allow to make direct comparisons, enabling progress to be monitored, i.e., the advances and setbacks of the performance of these web services.

†ミ INDICADORES DE ACESSO AO WEB SERVICE
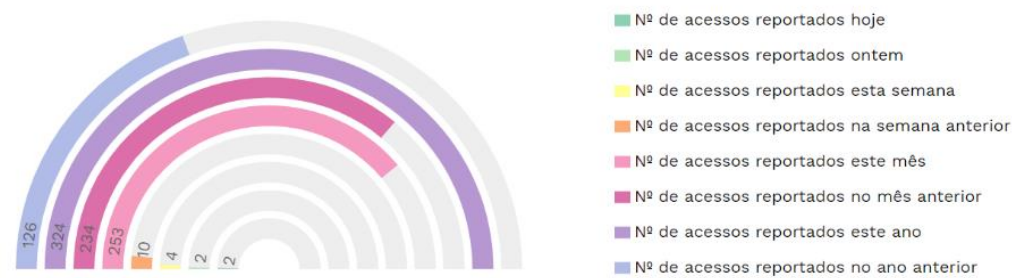

Fig. 4. Indicators related to the number of accesses to a web service by specific dates.

Tables Module. As the name suggests, displays information about the web services activities in the form of tables. The information displayed concern the accesses to the web service and the errors occurred. These tables are very interactive and allow not only to filter the table entries, either by typing in the free search filter or by selecting the interest values, as well as sorting those entries in ascending or descending order according to the fields that the user desires.

\subsection{Demonstration and Evaluation}

The demonstration stage aims to demonstrate the use of the artifact to solve one or more instances of the problem. At this point, the application must be submitted to specific tests in the hospital environment, using different web services and different contexts. The demonstration is only possible, after the successful implementation of the web application in the hospital environment.

In Information Technology (IT), any type of development project should be submitted to a set of tests and evaluations before being made available. The evaluation stage corresponds to the process of evaluating whether the developed solution solves the problem at hand and meets the requirements and specifications initially defined. Accordingly, after developing the web application, several tests were performed with information from different web services to verify and demonstrate its accuracy and completeness, allowing the identification and correction of errors. After considering the application stable and reliable, it is essential to test the solution in the hospital environment in order to correct eventual errors or inconsistencies and, consequently, improve the overall performance of the web application. This is the next step to per- 
form in the development process of the platform, since the web application must be fully integrated in the hospital environment.

\subsection{Communication}

The present work was the object of study of a master thesis in the area of medical informatics and culminated with the publication of the respective dissertation. Furthermore, the contributions of this effort were disclosed in several academic lectures at the University of Minho.

\section{Discussion of the Results}

The solution proposed in this study provides a dynamic, interactive, and user-friendly platform which allows key management staff to consult and manage the web services performance in order to keep track of the hospital's operations and study the processes behavior, optimizing them and improving its operational performance. The main benefits of the adoption of this monitoring platform were:

- Display of key information in a summarized and objective way through a simple and intuitive interface;

- Provision of instant access to relevant information about the web services activity and greater visibility into the information required to make critical decisions;

- Monitorization of hospital's web services and the study of processes behavior to optimize them and improve its operational performance;

- Maintenance of the web services behavior and prevention of unnecessary failures;

- The monitoring platform is scalable and easy to maintain, as its architecture is of easy maintenance and the expansion of its functionalities is very simple;

- By using modern, innovative and efficient technologies, the CHP is able to follow the technological advances, which offers a competitive advantage and brings benefits in terms of system usability. For example, the use of ReactJS brings several rewards in terms of system performance, of which stands out a fast rendering due to the existence of a virtual DOM and the ability to reuse and combine components.

\subsection{Proof of Concept}

After completing the technical development of the solution proposed as a resolution to the problems identified in the scope of this study, a Proof of Concept (PoC) was carried out to prove the viability and usefulness of the developed solution. In addition to identifying potential failures or errors in the developed solution, the use of a PoC allows to verify if the solution satisfies the requirements and objectives for which it was initially designed. Thus, a SWOT analysis was carried out to briefly define the strengths and weaknesses (internal factors) of the resulting product, as well as the opportunities and threats that it is exposed (external factors). The results of this analysis are shown in Table 1. 
Table 1. SWOT analysis

\begin{tabular}{|c|c|}
\hline Strengths & Weaknesses \\
\hline $\begin{array}{l}\text { - High usability: intuitive and user- } \\
\text { friendly tool; } \\
\text { - High scalability; } \\
\text { - Centralization of different infor- } \\
\text { mation and functionalities concerning } \\
\text { the web services performance in a } \\
\text { summarized and objective way. } \\
\text { - Web application easily adaptable to } \\
\text { different health institutions that may } \\
\text { need this type of clinical tool. }\end{array}$ & $\begin{array}{l}\text { - Requires constant connection to the } \\
\text { hospital internal network; } \\
\text { - Runs slower, when the requests for } \\
\text { information involve a high amount of } \\
\text { data; }\end{array}$ \\
\hline $\begin{array}{l}\text { Opportunities } \\
\text { - Growing demand of health units to } \\
\text { control the performance of services } \\
\text { that occur in background that are not } \\
\text { directly related with patient care; } \\
\text { - Need to control the occurrence of } \\
\text { errors and enable a faster response ac- } \\
\text { tion; } \\
\text { - Increase the quality and efficiency of } \\
\text { healthcare using new technologies. }\end{array}$ & $\begin{array}{l}\text { Threats } \\
\text { - Structural failures or changes in the } \\
\text { databases that "feed" the platform may } \\
\text { jeopardize its main functions; } \\
\text { - Problems with network connectivity to } \\
\text { the hospital intranet; } \\
\text { - Lack of interest or commitment in the } \\
\text { project. }\end{array}$ \\
\hline
\end{tabular}

\section{Conclusions}

An effective monitoring process allows to characterize a system and recognize its vulnerabilities and threats in order to determine the likelihood of a failure. The solution presented addresses this need by monitoring key information concerning the activity of numerous web services of a Portuguese Hospital. Modern and up to date technologies were adopted to create an intuitive and easy to interact with platform. The use of contemporary technologies, like React, helped in building a user interface with an attractive and pleasant design that makes the user experience simpler.

Accordingly, the execution of this case study culminated in the elaboration of an area restricted to the hospital's key management staff with live and interactive dashboards, including charts, tables, statistics, and, also, performance indicators, so that they can consult and manage the web services performance allowing greater control of its operation. Consequently, and since it also allows to observe in a timely manner the occurrence of errors or inconsistencies, it helps to identify and eliminate root causes of inefficiency and poor performance. This verification aims to optimize various functions and procedures as well as to ensure the web services functioning and efficiency. Ultimately, this streamlined approach enables and empowers executives 
and managers to check performance quickly, for an up-to-date glance at where things are going well and where improvements need to be made thus improving administrative and, consequently, clinical decision making which generates positive repercussions in the quality of the offered services and more so maximizes the financial returns. Accordingly, the use of innovative technologies and the new functionalities offered by the monitoring platform have enhanced the hospital modernization and improved the health care delivery.

As future work, new forms to increase the loading of the web application can be sought and studied. Furthermore, adapting the monitoring platform to be used in mobile devices could be highly beneficial. Last but not least, it would be significant to find new ways to raise the level of data security and confidentiality.

\section{Acknowledgements}

This work has been supported by FCT - Fundação para a Ciência e Tecnologia within the Project Scope: UID/CEC/00319/2019.

\section{References}

1. Haux, R., Ammenwerth, E., Winter, A., \& Brigl, B.: Strategic information management in hospitals: an introduction to hospital information systems. Springer Science \& Business Media (2004).

2. Buckingham, R. A., Hirschheim, R. A., Land, F. F., \& Tully, C. J.: Information systems education: recommendations and implementation. Cambridge University Press (1986).

3. Avison, D. E., \& Wood-Harper, A. T.: Multiview - An Exploration in Information Systems Development. Australian Computer Journal, 18(4), 174-179 (1986).

4. Laudon, K. C., \& Laudon, J. P.: Management information systems: managing the digital firm. Pearson (2018).

5. Davis, G. B., \& Olson, M. H.: Management information systems: conceptual foundations, structure, and development. McGraw-Hill, Inc. (1984).

6. Bonney, W.: Applicability of business intelligence in electronic health record. ProcediaSocial and Behavioral Sciences, 73, 257-262 (2013).

7. Buntin, M. B., Burke, M. F., Hoaglin, M. C., \& Blumenthal, D.: The benefits of health information technology: a review of the recent literature shows predominantly positive results. Health affairs, 30(3), 464-471 (2011).

8. Lee, J., McCullough, J. S., \& Town, R. J.: The impact of health information technology on hospital productivity. The RAND Journal of Economics, 44(3), 545-568 (2013).

9. Chen, R. F., \& Hsiao, J. L.: An investigation on physicians' acceptance of hospital information systems: a case study. International journal of medical informatics, 81(12), 810820 (2012).

10. Peffers, K., Tuunanen, T., Rothenberger, M. A., \& Chatterjee, S.: A design science research methodology for information systems research. Journal of management information systems, 24(3), 45-77 (2007). 